\title{
Health School-based Mindfulness Interventions for Improving Mental Health: A Systematic Review and Thematic Synthesis of Qualitative Studies
}

\author{
Supakyada Sapthiang ${ }^{1} \cdot$ William Van Gordon $\mathbb{1}^{2} \cdot$ Edo Shonin ${ }^{3}$
}

Published online: 5 June 2019

(c) The Author(s) 2019

\begin{abstract}
Objectives School-based mindfulness interventions have recently shown promise for treating and preventing mental health issues in young people. However, the literature lacks a high-level perspective of the impact of mindfulness on young people's mental health according to their own first-hand accounts. Therefore, the objective of this study was to conduct the first systematic review and thematic synthesis to rigorously evaluate the qualitative evidence pertaining to students' experiences of school-based MBIs.

Methods The following electronic databases were searched for qualitative school-based mindfulness intervention papers published up until the end of March 2019: PubMed, Web of Science, Scopus, ProQuest, and Google Scholar. An assessment of study quality was undertaken using the Critical Appraisal Skills Programme qualitative checklist.

Results The initial literature search returned 4102 papers and seven studies met all of the inclusion criteria. The thematic synthesis identified four major themes of (i) using attentional processes to regulate emotions and cognitions, (ii) stress reduction, (iii) improved coping and social skills, and (iv) calming and/or relaxation.

Conclusions Findings show that school-based MBIs are experienced by students as having a range of benefits to mental health, including in both preventative and treatment contexts. However, efforts should be made to improve methodological quality, including taking steps to minimise recall bias and provide a greater degree of transparency regarding how students are selected to attend qualitative interviews or focus groups.
\end{abstract}

Keywords Mindfulness $\cdot$ Mindfulness-based interventions $\cdot$ Schools $\cdot$ School-based interventions $\cdot$ Mental health $\cdot$ Public mental health

Approximately $10 \%$ of young people aged 5-16 years suffer from mental illness and half of all mental illnesses commence prior to the age of 14 (World Health Organization [WHO], N.D.). Risk factors for mental illness in young people include (for example) low socioeconomic status, social deprivation, adverse childhood experiences, pressures

William Van Gordon

w.vangordon@derby.ac.uk

1 Public Health Department, University of Essex, Grace Street, Leeds, UK

2 Human Sciences Research Centre, University of Derby, Kedleston Road, Derby, Derbyshire DE22 1GB, UK

3 Awake to Wisdom Centre for Meditation and Mindfulness Research, Ragusa, Italy relating to academic progression, and lack of family or community-based support (Broderick and Metz 2009; Sapthiang et al. 2019a; Van Gordon et al. 2019; WHO 2012). Stressors can also arise because of the physical, social, and cognitive-affective changes associated with child and youth transition. Mental illness during childhood and adolescence is linked to somatic health problems such as obesity, academic underachievement, delinquency, lower employment prospects, and psychiatric problems during adulthood (Dray et al. 2017; WHO 2012).

In addition to seeking to enhance mental illness treatment approaches for young people, there is growing awareness of the benefits of cultivating resiliency and protective traits (Sapthiang et al. 2019b; Schonert-Reichl and Lawlor 2010; Windle 2011). Accordingly, the UK government has made mental health promotion a priority area and has emphasised the important role of schools for improving young people's 
mental health (Sapthiang et al. 2018). Indeed, since most children spend a considerable amount of time at school, it is a viable setting for cultivating protective traits (e.g., emotional literacy, coping skills) (Van Gordon et al. 2019). This is in line with Rose's (1992) prevention paradox principle in which prevention targeted at low-risk individuals can be more effective at improving disease burden versus targeting high-risk individuals. Furthermore, in addition to the logistical ease of administering interventions to a collective group of students (Dray et al. 2017), delivering interventions as a whole class activity can help reduce stigma, social comparison, and inequality in accessing the intervention (Kuyken et al. 2013).

A category of intervention known as mindfulness-based interventions (MBIs) has recently shown promise in treatment and preventative contexts with school-aged young people (Sapthiang et al. 2018). The practice of mindfulness derives from Buddhist meditation and involves maintaining an active awareness of experienced phenomena on a moment-by-moment basis (Van Gordon et al. 2015). A systematic review of quantitative studies of mindfulness delivered to young people in school settings $(N=28$ included studies) demonstrated improvements in mental health variables such as depression, anxiety, rumination, behavioural distress, hostility, and aggression (Felver et al. 2016). The same systematic review also demonstrated increases in young people's resilience to mental illness through improvements in (for example) emotion regulation, attention skills, social skills, optimism, social-emotional competence, coping skills, and mindfulness (Felver et al. 2016).

However, according to Bannirchelvam et al. (2017), in addition to understanding and evaluating the evidence amassed through the aforementioned quantitative studies of mindfulness that have been conducted 'on' young people, it is essential to appraise and synthesise the evidence of qualitative mindfulness studies that have been conducted 'with' young people. Despite this, a systematic review of such qualitative studies has not been conducted to date, and the literature thus lacks a high-level perspective of the impact of mindfulness on young people's mental health according to their own first-hand accounts. Given that research supports young people's capability to elucidate and be experts of their experiential perspectives (Christensen and James 2000), a study reviewing such evidence is particularly needed. Furthermore, in light of growing evidence supporting the role of schools in adopting a public health approach toward building resilience and addressing mental health issues in young people, it would be helpful to determine what key themes underlie school students' lived experiences of participating in school-based MBIs.

Thus, the objective of this study was to conduct the first systematic review to rigorously appraise and synthesise the qualitative evidence pertaining to students' experiences of school-based MBIs. The extended version of the Population, Intervention, Control, and Outcomes (PICO; Boland et al. 2013) format was used to help identify the following research question: What key themes relevant to mental health underlie and/or reflect young people's experiences of participating in school-based MBIs? The Preferred Reporting Items for Systematic Reviews and Meta-analysis (PRISMA; McInnes et al. 2018) guidelines were followed were appropriate.

\section{Method}

\section{Literature Search}

Paper titles, abstracts and keywords were searched using the following search terms and criteria: Mindful* AND School*, OR Education*, OR Student* OR Youth OR Young OR Child* OR Adolecen*. In order to avoid overlooking qualitative components embedded within quantitative studies, search terms specifically relating to qualitative data collection or analytical techniques were not employed. The aforementioned search terms were applied to the following electronic databases for all papers published up until the end of March 2019: PubMed, Web of Science, Scopus, ProQuest, and Google Scholar. Reference lists of relevant studies and review papers were also searched.

\section{Selection Criteria and Data Extraction}

In order to be included in the systematic review, a study had to (i) involve the delivery of an MBI (whether a single session or lasting for several months), (ii) involve students attending either primary or secondary education, (iii) employ intervention delivery linked to a school setting (i.e., whether delivered at school or involving students referred by a school), (iv) utilise qualitative data collection and analytical techniques, (v) report findings relevant to mental health, and (vi) be published in a peer-reviewed journal in English language. Papers were excluded if they (i) did not elicit responses directly from students, (ii) did not include an intervention specifically designated as being focussed on mindfulness (e.g., studies based on "yoga" or unspecified forms of meditation), (iii) only reported findings relevant to academic performance, and (iv) were theoretical or review papers (see Table 1 for alignment of inclusion and exclusion criteria with PICO elements). Studies not published in peerreviewed journals were excluded on the basis that publication in a refereed journal should provide at least a minimum level of assurance in respect of a study's methodological approach, ethical propriety, reporting standards, and general credibility (Shonin et al. 2015). 
Table 1 Extended PICO for this systematic review

Review question What key themes relevant to mental health underlie and/or reflect young people's experiences of participating in schoolbased MBIs?

\begin{tabular}{lll} 
& Inclusion criteria & Exclusion criteria \\
\cline { 2 - 3 } $\begin{array}{ll}\text { Population } \\
\text { Intervention }\end{array}$ & Young people of school-going age & Not attending primary or secondary school \\
Comparator & Not applicable & An intervention not specifically designated as being focussed on mindfulness \\
Outcomes & Any themes relevant to mental health & Not applicable \\
Study design & Qualitative intervention study & Educational performance \\
& & Quantitative studies (excluding those with an embedded qualitative arm), review \\
Other & Published in a peer-reviewed academic & Did not elicit responses directly from students
\end{tabular}

Abstracts were screened and a paper was retrieved and analysed for study eligibility if it appeared to include relevant data or information. The PRISMA guidelines (McInnes et al. 2018) were used to inform the choice of extracted data items, which included sample size, sample description (e.g., age range, clinical/non-clinical, country of intervention delivery), intervention description (e.g., course length, session frequency, session duration, key techniques employed, etc.), details of intervention facilitator, qualitative data collection technique, qualitative analytical technique, and mental health-relevant themes.

\section{Assessment of Study Quality}

An assessment of study quality was undertaken using the Critical Appraisal Skills Programme (CASP) qualitative checklist (CASP 2018). Tools such as the CASP qualitative checklist (that includes ten questions such as: "Was the recruitment strategy appropriate to the aims of the research?" "Was the data collected in a way that addressed the research issue?") provide a transparency of research methods which permits an evaluation of the credibility and transferability of the results of the primary study (Morton et al. 2010). However, the CASP assessment was not used as a basis for excluding or weighting any of the eligible studies.

\section{Data Synthesis}

A thematic synthesis was undertaken to elicit a rigorous high-level analytical abstraction of common elements and themes across eligible qualitative studies (Thomas et al. 2008). To allow the analysis of a substantive literature in which individual studies represented a wide range of study aims, the thematic synthesis focussed on identifying common underlying themes relevant to school students' mental health. Verbatim participant extracts taken from the results section of each eligible study were inputted into NVivo
11 software for storing, coding, and data searching (Morton et al. 2010; Thomas et al. 2008). The thematic synthesis employed phases of (i) coding participant extracts on a lineby-line basis for each eligible study, (ii) forming descriptive themes, and (iii) generating analytical themes (Thomas et al. 2008). Studies were read several times to confirm that all of the extracts of students' perspectives were captured (Morton et al. 2010). Participant perspectives were then assessed for convergence in order to formulate the final thematic structure (Lucas et al. 2007).

\section{Results}

\section{Search Results}

The initial literature search returned 4102 papers. After the review of the papers' abstracts, 4020 studies were deemed ineligible according to the aforementioned eligibility criteria. Following a full-text review of the remaining 82 papers, seven studies met all of the inclusion criteria and were subjected to an in-depth review and appraisal. The PRISMA flow diagram outlining the paper selection process is shown in Fig. 1.

\section{Primary Reasons for Exclusion}

Of the 82 papers subjected to a full-text review, the main reasons for ineligibility were because the study (i) did not involve young people attending a primary or secondary school (e.g., Chrisman et al. 2009; Monshat et al. 2013), (ii) used an intervention that was not principally based on and/ or described as mindfulness (e.g., Campion and Rocco 2009; Conboy et al. 2013), (iii) primarily relied on quantitative assessments with insufficient detail to determine the qualitative data collection process, qualitative analytical technique, and/or key themes identified (e.g., Broderick and Metz 2009; Metz et al. 2013; Tharaldsen 2012; Wall 2005), 
Fig. 1 PRISMA flow diagram of the paper selection process

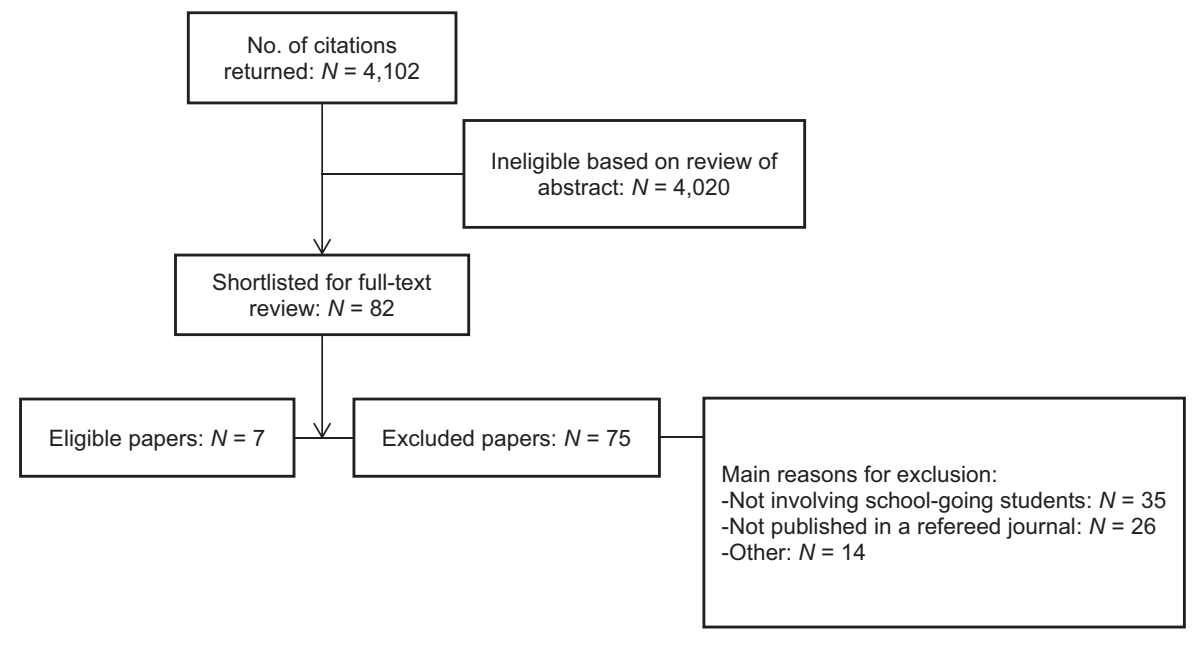

(iv) was not described as being a school-based intervention (e.g., Milligan et al. 2015), (v) did not directly elicit students' perspectives (e.g., Capel 2012), (vi) was not published in English language (e.g., Jin 2016), and (vii) was not published in a peer-reviewed journal.

\section{Overview of Study Characteristics}

The combined sample size of the seven eligible studies was $n=251(M=35.86, S D=29.40)$. All seven eligible studies were published since 2014. Participants ranged in age between 7 and 18 years, and were based in Ireland (2 studies), US (2 studies), England, Australia, and Canada. Four studies were based in schools serving socioeconomically disadvantaged areas, and three studies specifically targeted students with mental health and/or behavioural issues (see Table 2 for a description of included studies).

Four studies used interviews (semi-structured interviews in the case of three studies) as the qualitative data collection technique and the remaining three studies used focus groups. Five studies used thematic analysis as the qualitative analytical approach. The duration of the MBI ranged from 5 to 20 weeks (duration not specified in Tunney et al. 2017). The range of MBI facilitators included registered psychologists, yoga instructors, school teachers and assistant teachers, mental health therapists, and study researchers (intervention facilitator details not provided in Costello and Lawler 2014).

\section{Assessment of Study Quality}

The assessment of study quality using the CASP checklist (2018) indicated variability in methodological robustness across the seven eligible studies. All of the eligible studies reported the sample size, provided a description of the sample, and included participant quotations. There were also no issues across the eligible studies in respect of providing a clear statement of aims, using appropriate qualitative methodology, using a research design suitable for the research aims, adequately considering ethical issues, providing a clear statement of findings, and performing value-adding research (CASP items 1-3, 7, 9, and 10). However, as shown in Table 3 , there were a number of methodological issues (between 2 and 4 'No' responses to CASP items per study) relating to ambiguous or unsuitable recruitment strategy, inappropriate data collection, lack of consideration of the relationship between participants and researcher, and poorly-defined or inappropriate data analytical strategy (CASP items 4-6, and 8).

\section{Synthesis}

Four major themes emerged as being relevant to students' mental health following participation in a school-based MBI (i) using attentional processes to regulate emotions and cognitions, (ii) stress reduction, (iii) improved coping and social skills, and (iv) calming and/or relaxation (see Table 4). The first theme referred to students' experience that mindful attention helped them to foster a greater awareness of and/or let go of emotions and cognitive processes. The second theme reflected students' experience of reductions in stress as well as an appreciation of the utility of mindfulness for stress reduction. The third theme referred to students' experience of enhanced coping and resilience skills as well as improvements in social skills. The fourth theme reflected students' experiences of both psychological and somatic forms of relaxation elicited through mindfulness practice.

Challenges and acceptability issues (e.g., misconceptions concerning mindfulness) were not targeted as part of the current systematic review but in any event, such issues would not have constituted a common theme as they were identified in only three of the seven eligible studies (i.e., Bannirchelvam et al. 2017; Dariotis et al. 2017; McGeechan et al. 2019). 


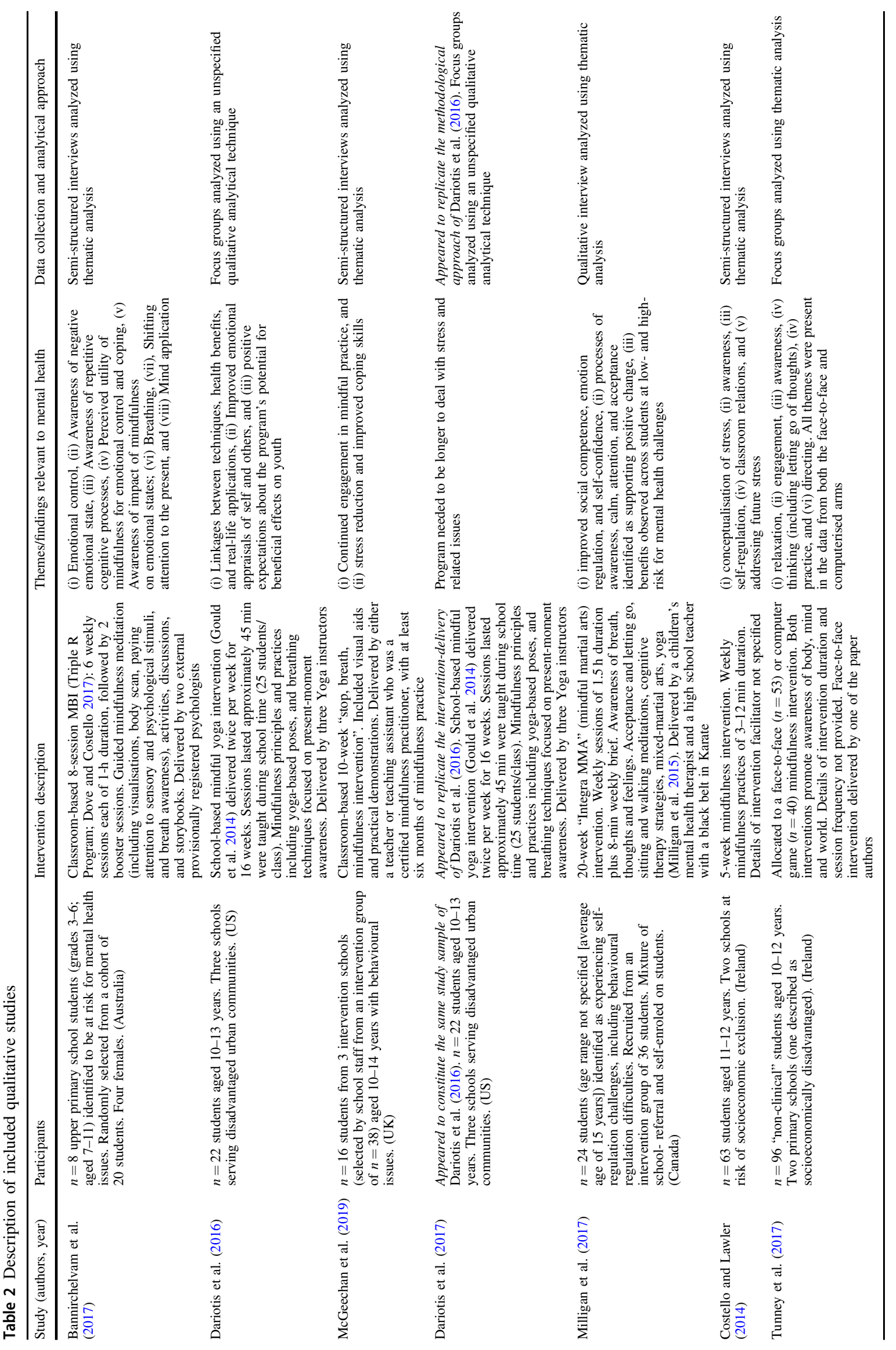




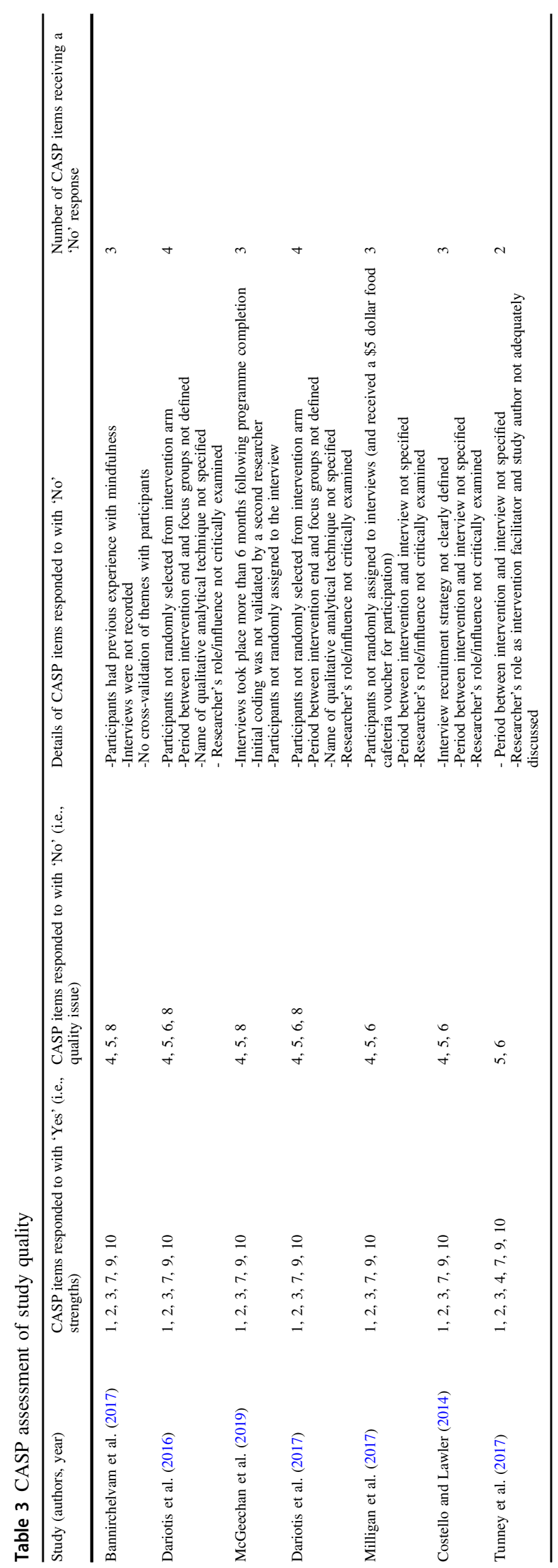

\section{Discussion}

The present systematic review evaluated the qualitative evidence relevant to mental health arising from students' participation in school-based MBIs. Seven studies-which had all been published since 2014 - met the eligibility criteria. A thematic synthesis included as part of the systematic review identified four major themes: using attentional processes to regulate emotions and cognitions, stress reduction, improved coping and social skills, and calming and/or relaxation.

In the context of the first master theme, participants reported how mindful attention helped them to observe their thoughts and feelings and respond rather than habitually react to a given stimulus. This is consistent with the consensus view that mindfulness involves focussing attention on moment-to-moment sensory and psychological experiences in an open and accepting manner (Chapman and Van Gordon 2018). This greater awareness of cognitive-affective processes appeared to play an important role in improving emotion regulation.

The school-based MBIs included in the present review also appeared to augment students' capacity for coping with the challenges associated with child and adolescent transition (i.e., the third Master theme to emerge from the dataset). Indeed, elsewhere researchers have highlighted both the immediate and preventative applications associated with learning mindfulness prior to adulthood (e.g., Sapthiang et al. 2018). In the present systematic review, improvements in coping skills were also reported to positively impact on social skills. This finding is noteworthy given the correlation between mental illness and social deprivation (WHO 2012).

Mindfulness was also understood by participants to be a valuable means of reducing stress as well as fostering calm and relaxation (i.e., the second and fourth master themes). Given that stress is a known risk factors for mental illness in young people (WHO 2012), techniques that can lower school students' stress levels will obviously have applications in a range of applied contexts. Furthermore, while a link between relaxation and stress reduction was not reported in every study included in this systematic review, mindfulness has been shown to induce relaxation through reduced autonomic arousal that, in turn, helps to dissipate accumulated stress (Shonin et al. 2015).

While this systematic review appears to support the utility of MBIs as school-based interventions for improving mental health, findings should be viewed in light of the limitations of the seven included studies, as well as the limitations of the systematic review itself. An assessment of methodological quality using the CASP (2018) qualitative checklist indicated a number of quality issues pertaining to the eligible studies. Of particular note was a lack of 
Table 4 Distribution of master themes

\begin{tabular}{|c|c|c|c|c|}
\hline Study (authors, year) & $\begin{array}{l}\text { Theme 1: using attentional processes to } \\
\text { regulate emotions and/or cognitions }\end{array}$ & $\begin{array}{l}\text { Theme 2: stress } \\
\text { reduction }\end{array}$ & $\begin{array}{l}\text { Theme 3: improved coping } \\
\text { and social skills }\end{array}$ & $\begin{array}{l}\text { Theme } 4 \text { : calming and/ } \\
\text { or relaxation }\end{array}$ \\
\hline $\begin{array}{l}\text { Bannirchelvam et al. } \\
\text { (2017) }\end{array}$ & Yes & Yes & Yes & Yes \\
\hline Dariotis et al. (2016) & Yes & Yes & Yes & Yes \\
\hline McGeechan et al. (2019) & No & Yes & Yes & Yes \\
\hline Dariotis et al. (2017) & No & Yes & No & No \\
\hline Milligan et al. (2017) & Yes & Yes & Yes & Yes \\
\hline $\begin{array}{l}\text { Costello and Lawler } \\
\text { (2014) }\end{array}$ & Yes & Yes & Yes & Yes \\
\hline Tunney et al. (2017) & Yes & No & No & Yes \\
\hline
\end{tabular}

reporting of the period between intervention end and data collection, which made it difficult to assess whether recall bias may have influenced the findings. A further quality issue was that biases may have been present when selecting which students should attend the interview or focus group, as in most instances random assignment (or a convincing alternative approach) was not employed.

Key limitations relevant to the present systematic review were that despite incorporating the experiences of 251 school-going students based in five countries, unpublished and non-English language studies were excluded. Furthermore, by restricting the focus to school-based interventions, studies of young people receiving mindfulness training not linked to a school were also not included. Thus, it is possible that some potentially useful evidence may have been excluded.

A key implication based on the accounts of the students included in this systematic review is that school-based MBIs are experienced by students as having a range of benefits to mental health, including in both preventative and treatment contexts. However, an important future direction for school-based qualitative research is to improve methodological quality, including taking steps to minimise recall bias and provide a greater degree of transparency regarding how students are selected to attend qualitative interviews or focus groups.

Funding No financial support was received for this study.

Author Contributions All authors were involved in the conception and writing of the paper. Furthermore, we confirm that all authors are responsible for all contents of the paper and had authority over manuscript preparation and the decision to submit the manuscript for publication.

\section{Compliance with Ethical Standards}

Conflict of Interest All authors declare that they have no conflict of interest apart from W.V.G. who delivers consulting and training relating to using contemplative approaches in applied settings. W.V.G. also receives royalties from books relating to contemplative practice.

Publisher's note: Springer Nature remains neutral with regard to jurisdictional claims in published maps and institutional affiliations.

Open Access This article is distributed under the terms of the Creative Commons Attribution 4.0 International License (http://crea tivecommons.org/licenses/by/4.0/), which permits unrestricted use, distribution, and reproduction in any medium, provided you give appropriate credit to the original author(s) and the source, provide a link to the Creative Commons license, and indicate if changes were made.

\section{References}

Bannirchelvam, B., Bell, K. L., \& Costello, S. (2017). A qualitative exploration of primary school students' experience and utilisation of mindfulness. Contemporary School Psychology, 21, 304-316. https://doi.org/10.1007/s40688-017-0141-2.

Boland, A., Cherry, G., \& Dickson, R. (2013). Doing a systematic review. London: Sage.

Broderick, P. C., \& Metz, S. (2009). Learning to BREATHE: a pilot trial of a mindfulness curriculum for adolescents. Advances in School Mental Health Promotion, 2, 35-46.

Campion, J., \& Rocco, S. (2009). Minding the mind: the effects and potential of a school-based meditation programme for mental health promotion. Advances in School Mental Health Promotion, 2, 47-55. https://doi.org/10.1080/1754730X.2009.9715697.

Capel, C. M. (2012). Mindlessness/mindfulness, classroom practices and quality of early childhood education: an auto-ethnographic and intrinsic case research. International Journal of Quality and Reliability Management, 29, 666-680. https://doi.org/10.1108/ 02656711211245656.

Chapman, C., \& Van Gordon, W. (2018). Effects of a brief online mindfulness intervention on mindfulness, psychological distress and parenting stress in preschool parents. Mindfulness and Compassion, 3, 55-70.

Chrisman, J. A., Christopher, J. C., \& Lichtenstein, S. J. (2009). Qigong as a mindfulness practice for counselling students: a qualitative study. Journal of Humanistic Psychology, 49, 236-257. https://doi.org/10.1177/0022167808327750.

Christensen, P. \& James, A. (2000). Research with children: perspectives and practices. https://www.amazon.co.uk/ResearchChildren-Perspectives-Pia-Christensen/dp/0415416841. Accessed 14 Apr 2019. 
Conboy, L. A., Noggle, J. J., Frey, J. L., Kudesia, R. S., \& Khalsa, S. B. S. (2013). Qualitative evaluation of a high school yoga program: feasibility and perceived benefits. Explore, 9, 171-180. https://doi.org/10.1016/j.explore.2013.02.001.

Costello, E., \& Lawler, M. (2014). An exploratory study of the effects of mindfulness on perceived levels of stress among schoolchildren from lower socioeconomic backgrounds. The International Journal of Emotional Education, 6, 21-39.

Critical Appraisal Skills Programme (CASP). (N.D.). Checklist: 10 questions to help you make sense of a qualitative research. https:// mail.google.com/mail/u/0/?tab=rm\#inbox/FMfcgxw

CgLnvRLJwWsfDwrKJfTwqGqKm?projector=1\&messagePa rtId=0.4. Accessed 14 Apr 2019.

Critical Appraisal Skills Programme. (2018). CASP qualitative checklist. https://casp-uk.net/casp-tools-checklists/. Accessed 15 Apr 2019.

Dariotis, J. K., Mirabal-Beltran, R., Cluxton-Keller, F., Gould, L. F., Greenberg, M. T., \& Mendelson, T. (2017). A qualitative exploration of implementation factors in a school-based mindfulness and yoga program: lessons learned from students and teachers. Psychology in the Schools, 54, 53-69. https://doi.org/ 10.1002/pits.21979.

Dariotis, J. K., Mirabal-Beltran, R., Cluxton-Keller, F., Gould, L. F., Greenberg, M. T., \& Mendelson, T. (2016). A qualitative evaluation of student learning and skills use in a school-based mindfulness and yoga program. Mindfulness, 7, 76-89. https:// doi.org/10.1007/s12671-015-0463-y.

Dove, C. \& Costello, S. (2017). Supporting emotional well-being in schools: a pilot study into the efficacy of a mindfulness-based group intervention on anxious and depressive symptoms in children. Advances in Mental Health: Promotion, Prevention, and Early Intervention, 1-11. https://doi.org/10.1080/18387357. 2016.1275717

Dray, J., Bowman, J., Campbell, E., Freund, M., Wolfenden, L., Hodder, R. K., \& Wiggers, J. (2017). Systematic review of universal resilience-focused interventions targeting child and adolescent mental health in the school setting. Journal of the American Academy of Child and Adolescent Psychiatry, 56, 813-824. https://doi.org/10.1016/j.jaac.2017.07.780.

Felver, J. C., Hoyos, C., Tezanos, K., \& Singh, N. (2016). A systematic review of mindfulness-based interventions for youth in school settings. Mindfulness, 7, 34-45. https://doi.org/10.1007/ s12671-015-0389-4.

Gould, L. F., Mendelson, T., Dariotis, J. K., Ancona, M., Smith, A. S., Gonzalez, A. A., \& Greenberg, M. T. (2014). Assessing fidelity of core components in a mindfulness and yoga intervention for urban youth: applying the CORE process. New Directions for Youth Development, 142, 59-81. https://doi.org/10.1002/yd.20097.

Jin, Y. (2016). A qualitative study about effects and risks of the mindfulness program at the girls' training school. Japanese Journal of Mindfulness, 1, 28-40.

Kuyken, W., et al. (2013). Effectiveness of the mindfulness in schools programme: non-randomised controlled feasibility study. British Journal of Psychiatry, 203, 126e131.

Lucas, P. J., Baird, J., Arai, L., Law, C., \& Roberts, H. M. (2007). Worked examples of alternative methods for the synthesis of qualitative and quantitative research in systematic reviews. BMC Medical Research Methodology, 15, 4 https://doi.org/10.1186/ 1471-2288-7-4.

McGeechan, G., Richardson, C., Wilson, L., Allan, K., \& NewburyBirch, D. (2019). Qualitative exploration of a targeted schoolbased mindfulness course in England. Child and Adolescent Mental Health, 24, 154-160. https://doi.org/10.1111/camh.12288.

McInnes, M. D. F., Moher, D., Thombs, B. D., McGrath, T. A., Bossuyt, P. M., The PRISMA-DTA Group, \& Willis, B. H. (2018). Preferred reporting items for a systematic review and meta-analysis of diagnostic test accuracy studies: the PRISMA-
DTA statement. Journal of the American Medical Association, 319, 388-396. https://doi.org/10.1001/jama.2017.19163.

Metz, S. M., Frank, J. L., Reibel, D. K., Cantrell, T., \& Sanders, R. (2013). The effectiveness of the learning to BREATHE program on adolescent emotion regulation. Research in Human Development, 10, 252-272. https://doi.org/10.1080/15427609.2013.818488.

Milligan, K., et al. (2017). Integrating mindfulness into mixed martial arts training to enhance academic, social, and emotional outcomes for at-risk high school students: a qualitative exploration. Contemporary School Psychology, 21, 335-346. https://doi.org/ 10.1007/s40688-017-0142-1.

Milligan, K., Badali, P., \& Spiroiu, F. (2015). Using Integra mindfulness martial arts to address self-regulation challenges in youth with learning disabilities: a qualitative exploration. Journal of Child and Family Studies, 24, 562-575. https://doi.org/10.1007/ s10826-013-9868-1.

Monshat, K., et al. (2013). A conscious control over life and my emotions: mindfulness practice and healthy young people. A qualitative study. Journal of Adolescent Health, 52, 572-577. https://doi.org/10.1016/j.jadohealth.2012.09.008.

Morton, R. L., Tong, A., Howard, K., Snelling, P., \& Webster, C. (2010). The views of patients and carers in treatment decision making for chronic kidney disease: systematic review and thematic synthesis of qualitative studies. British Medical Journal, 340, c112. https://doi.org/10.1136/bmj.c112.

Rose, G. (1992). The strategy of preventive medicine. Cambridge: Oxford University Press.

Salmoirago-Blotcher, E., et al. (2018). Integrating mindfulness training in school health education to promote healthy behaviors in adolescents: feasibility and preliminary effects on exercise and dietary habits. Preventive Medicine Reports, 9, 92-95. https://doi. org/10.1016/j.pmedr.2018.01.

Sapthiang, S., Van Gordon, W., \& Shonin, E. (2018). Mindfulness in schools: a health promotion approach to improving adolescent mental health. International Journal of Mental Health and Addiction, 17, 112-119. https://doi.org/10.1007/s11469-018-0001-y.

Sapthiang, S., Van Gordon, W., Shonin, E., \& Griffiths, M. D. (2019a). The mental health needs of child and adolescent refugee and asylum seekers entering Europe. Social Health and Behavior, 2, 13-16. https://doi.org/10.4103/SHB.SHB_38_18.

Sapthiang, S., Van Gordon, W., Shonin, E., \& Griffiths, M. D. (2019b). Adolescent problem gambling requires population-level public health interventions. Addiction Research and Theory. https://doi.org/10.1080/16066359.2019.1590559.

Schonert-Reichl, K. A., \& Lawlor, M. S. (2010). The effects of a mindfulness-based education program on pre- and early adolescents' well-being and social and emotional competence. Mindfulness, 1, 137-151. https://doi.org/10.1007/s12671-010-0011-8.

Shonin, E., Van Gordon, W., \& Griffiths, M. D. (2015). Mindfulness in psychology: a breath of fresh air? The Psychologist, 28, 28-31.

Shonin, E., Van Gordon, W., Compare, A., Zangeneh, M., \& Griffiths, M. D. (2015). Buddhist-derived loving-kindness and compassion meditation for the treatment of psychopathology: a systematic review. Mindfulness, 6, 1161-1180. https://doi.org/10.1007/ s12671-014-0368-1.

Tharaldsen, K. (2012). Mindful coping for adolescents: beneficial or confusing. Advances in School Mental Health Promotion, 5 , 105-124. https://doi.org/10.1080/1754730X.2012.691814.

Thomas, J., \& Harden, A. (2008). Methods for the thematic synthesis of qualitative research in systematic reviews. BMC Medical Research Methodology, 8, 45 https://doi.org/10.1186/1471-2288-8-45.

Tunney, C., Cooney, P., Coyle, D., \& O’Reilly, G. (2017). Comparing young people's experience of technology-delivered V. face-to-face mindfulness and relaxation: two-armed qualitative focus group study. The British Journal of Psychiatry, 210, 284-289. https://doi. org/10.1192/bjp.bp.115.172783. 
Van Gordon, W., Sapthiang, S., Shonin, E., \& Griffiths, M. D. (2019). Mindfulness for addressing key public health concerns in young people: preventative applications and safety concerns. Education and Health, 37, 9-14.

Van Gordon, W., Shonin, E., \& Griffiths, M. D. (2015). Towards a second-generation of mindfulness-based interventions. Australia and New Zealand Journal of Psychiatry, 49, 591-593. https://doi. org/10.1177/0004867415577437.

Wall, R. B. (2005). Tai Chi and mindfulness-based stress reduction in a Boston public middle school. Journal of Paediatric Health Care, 19, 230-237. https://doi.org/10.1016/j.pedhc.2005.02.006.
Windle, G. (2011). What is resilience? A review and concept analysis Reviews in ClinicalGerontology, 21, 152-169. https://doi.org/10. 1017/S0959259810000420.

World Health Organization (2012). Mapping actions of nongovernmental organizations and other international development organizations. http://apps.who.int/iris/bitstream/handle/10665/44875/ 9789241503648_eng.pdf;jsessionid=C2E61168463EF95016D 60482AEEAAC91? sequence $=1$. Accessed 13 Apr 2019

World Health Organization. (N.D.) Child and adolescent mental health. http://www.who.int/mental_health/maternal-child/child_a dolescent/en/. Accessed 13 Apr 2019 